\title{
Anisotropic magnetic susceptibility of multiwalled carbon nanotubes
}

\author{
F. Tsui, ${ }^{\text {a) }}$ L. Jin, and O. Zhou \\ Department of Physics and Astronomy and Curriculum in Applied and Materials Sciences, \\ University of North Carolina, Chapel Hill, North Carolina 27599
}

(Received 28 September 1999; accepted for publication 20 January 2000)

\begin{abstract}
Magnetic susceptibility of partially aligned multiwalled carbon nanotubes has been studied using superconducting quantum interference device magnetometry. Partial alignment of multiwalled nanotubes was produced by uniaxially straining composites of carbon nanotubes embedded in polymer matrices. The degree of alignment was determined by $\mathrm{x}$-ray diffraction. The observed magnetic response is diamagnetic and anisotropic with the component along the nanotubes less diamagnetic than that of the perpendicular. The observed anisotropy is consistent with theoretical predictions, but it contradicts earlier experimental findings. (c) 2000 American Institute of Physics. [S0003-6951(00)04011-0]
\end{abstract}

Carbon nanotubes (CNT) exhibit a variety of unique properties. ${ }^{1}$ The presence of a large magnetic susceptibility with strong anisotropy is particularly interesting. ${ }^{2-7}$ While the observed large diamagnetism ${ }^{4-7}$ (with mass susceptibility at $300 \mathrm{~K}$ about $-10^{-5} \mathrm{emu} / \mathrm{g}$ ) is consistent with theoretical predictions, ${ }^{2,3}$ its anisotropy with a more diamagnetic component along the nanotubes ${ }^{4,6,7}$ (about $-10^{-6} \mathrm{emu} / \mathrm{g}$ more) is opposite to the predictions. ${ }^{2,3}$ The difference has not been resolved, owing to the lack of a systematic method to control and manipulate the alignment of nanotubes. Bulk susceptibility measurements to date have been performed using asgrown nanotube samples, whose alignment was not controlled nor determined quantitatively., ${ }^{4,6}$, Systematic and controlled magnetization experiments with aligned nanotubes have been very difficult. Recently, techniques have been developed to align multiwalled carbon nanotubes (MWNTs) by uniaxially stretching polymer-based MWNTs composites. ${ }^{8}$ This has made it possible to carry out studies of MWNTs with controlled alignment. In this letter we report a systematic study of bulk magnetic susceptibility of partially aligned MWNTs fabricated by this method.

Samples used in this study were produced by uniaxially strained composites of MWNT powder embedded in polymer matrices. ${ }^{8}$ It had been shown earlier that partial surface alignment, of nanotubes can be achieved locally by cutting the polymer/nanotube composite. ${ }^{9}$ Here, the MWNTs were synthesized by the arc-discharge method without any magnetic catalyst. ${ }^{10}$ A thermoplastic polymer was used as the matrix. Our transmission electron microscopy (TEM) measurements show that the MWNT powder contained $\sim 30 \%$ impurities, most of which were multishell nanoparticles, and no observable amount of graphitic materials. For the study, a series of composites with different nominal MWNT concentrations from 20 to $50 \mathrm{wt} \%$ were cast and subsequently stretched into mats to achieve partial alignment. The concentrations indicated in this letter are all nominal values. The alignment was characterized using $\mathrm{x}$-ray diffraction and TEM. ${ }^{8}$ The MWNT in as-cast composites exhibit no alignment and those in stretched mats show partial alignment

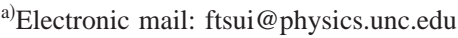

along the stretching direction. The quality of the alignment can be determined by azimuthal x-ray scans across the (002) reflection of MWNTs, which corresponds to the intershell spacing of MWNTs and carbon nanoparticles. As illustrated in Fig. 1 for a 50\% MWNT composite, a flat azimuthal intensity [Fig. 1(a)] indicates no alignment in the as-cast composite, and a pair of narrow azimuthal peaks $180^{\circ}$ apart [Fig. 1(b)] corresponds to alignment in the stretched sample. The shape of the azimuthal scan reflects the actual angular distribution of the MWNT with its width the width of the distribution. The integrated intensity under the curve, i.e., the azimuthal $\phi$ scan across (002), is proportional to the amount of aligned MWNTs, thus giving a quantitative measure of the alignment with respect to the isotropic background from unaligned materials. Details of the synthesis and structural quality were discussed elsewhere. ${ }^{8}$ In this research the measured azimuthal intensities were used as the angular distribution functions for deconvoluting the properties of a single nanotube. Stretched samples having a full width of about $40^{\circ}$ in their azimuthal scans were selected for the susceptibility study, and about $60 \%$ of MWNTs contained in these composites show partial alignment. Many batches of composites with a wide range of nominal MWNT compositions have been studied. Magnetic susceptibilities were measured using superconducting quantum interference device magnetometry in the temperature range between 5 and $300 \mathrm{~K}$ and field

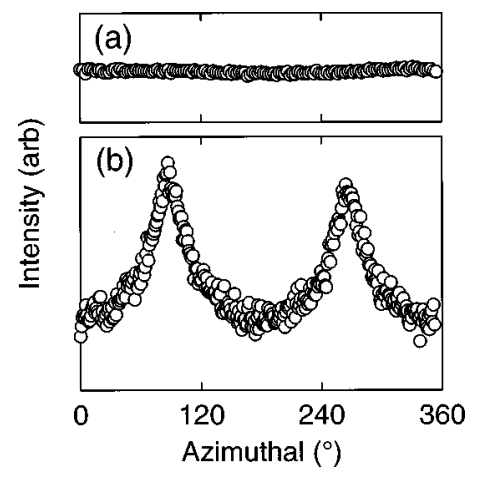

FIG. 1. Integrated x-ray intensity along the (002) reflection of the MWNT vs the azimuthal angle $\phi$ across the (002) reflection for (a) an as-cast 50 wt. \% MWNT/polymer composite, and (b) its partially aligned counterpart. 


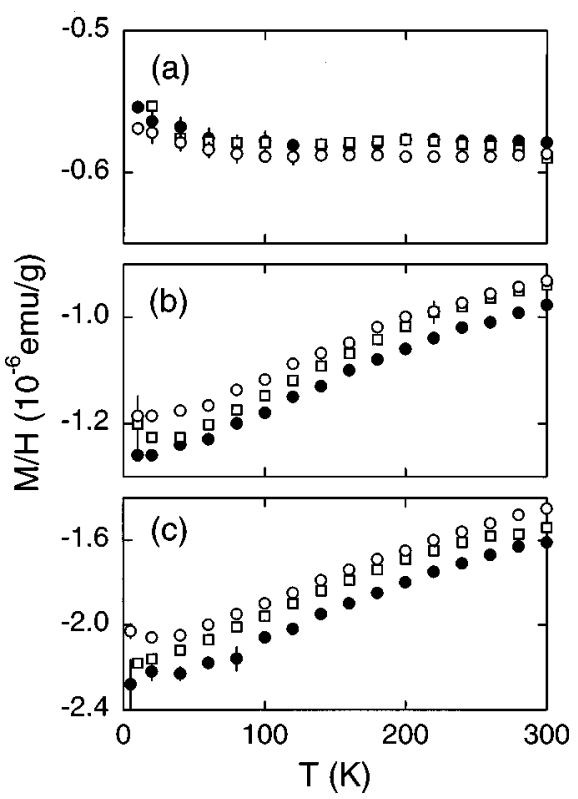

FIG. 2. Temperature dependent magnetic susceptibilities at $H=5 \mathrm{~T}$ along symmetry directions of (a) a pure polymer, (b) a 20 wt. \% MWNT composite, and (c) a 50 wt. \% MWNT composite. Open squares indicate the values for as-cast (random) composites, and circles are for stretched composites with open circles indicating the values along the stretched direction and closed circles indicating the perpendicular values.

range of $\pm 5 \mathrm{~T}$. Composite mats were suspended in the magnetometer by plastic straws. Magnetic responses were measured before and after the composite mats were stretched, and along and perpendicular to the stretched direction in order to determine the anisotropy. In order to determine the contribution of the polymer, pure polymer samples were made and stretched, and their magnetic responses measured.

Temperature dependent magnetic susceptibilities of several MWNTs/polymer composites at $5 \mathrm{~T}$ are shown in Fig. 2. The composites exhibit large diamagnetism, with the component along the nanotubes more paramagnetic [Figs. 2(b) and 2(c)]. The observed anisotropy is consistent with theoretical predictions, but opposite of earlier experimental results. ${ }^{4,6,7}$ The susceptibility of the pure polymer is diamagnetic and small, and it is nearly isotropic and temperature independent [Fig. 2(a)]. The values for MWNT composites exhibit strong temperature dependence, indicating that they are dominated by orbital contributions. The observed diamagnetic susceptibility and anisotropy increase as the MWNT concentration increases. This indicates that the values for pure MWNTs should be much larger, perhaps more than twice as large as that of the $50 \%$ composite. The observed susceptibility values for the as-cast samples are nearly the same as those averaged between the two orthogonal orientations for the respective stretched samples. This supports the notion that no intrinsic changes occur in the susceptibility as a result of stretching, as one would expect. In other words, the measured values for the as-cast samples correspond to the isotropic component of the susceptibility, and their differences with the values for the stretched samples along and perpendicular to the alignment direction represent the anisotropic component. The isotropic part contains responses from both unaligned MWNTs and nanoparticles, in addition to those of the polymer, but the anisotropic part arises only from the aligned MWNTs. We will return to this when we estimate

Downloaded 26 Nov 2002 to 152.2.6.144. Redistribution subject

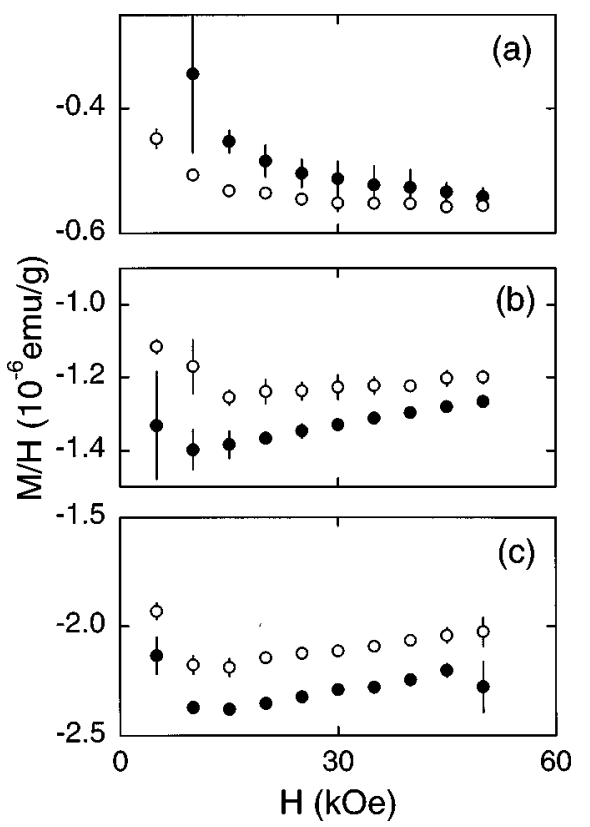

FIG. 3. Field dependent magnetic susceptibilities at $T=5 \mathrm{~K}$ along (open circles) and perpendicular to (closed circles) the stretched direction of (a) a pure polymer, (b) a $20 \mathrm{wt}$ \% MWNT composite, and (c) a $50 \mathrm{wt}$ \% MWNT composite. Lines are to guide the eyes.

the intrinsic susceptibility values of MWNTs.

Field dependent susceptibilities have been measured. The behavior at $5 \mathrm{~K}$ for several composites is shown in Fig. 3. The observed anisotropy for the composites is consistent with the temperature dependent behavior at $5 \mathrm{~T}$ discussed above. Within experimental uncertainty, the behavior for pure polymer is isotropic. The observed field dependence at low temperature is slightly nonlinear, and the dependences along and perpendicular to the alignment are comparable. The observed field dependence is similar to those reported earlier, ${ }^{4,6}$ but again, the observed anisotropy is the opposite of earlier results. ${ }^{4,6,7}$

In order to estimate the intrinsic susceptibility of the MWNTs, three factors have been considered and deconvoluted, and the results based on the method described here are shown in Fig. 4. First, the contributions from the polymer were eliminated by extrapolating the observed susceptibility for different nominal compositions at a given temperature to

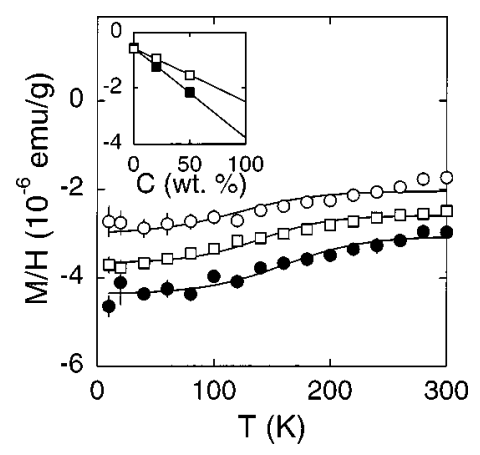

FIG. 4. Estimated magnetic susceptibility for fully aligned MWNTs. Squares correspond to the isotropic component, and circles represent the anisotropic values with the open circles the values along the tubes and closed circles the perpendicular values. Lines are to guide the eyes. Inset, susceptibility of as-cast composites vs MWNT concentration in wt. \% at 300 $\mathrm{K}$ (open squares) and $20 \mathrm{~K}$ (closed squares). Lines are linear fits to illustrate the extrapolation procedure described in the text. 
values at $100 \%$ MWNT, as illustrated in Fig. 4 inset. In this way, the isotropic $(M / H)_{\text {iso }}$ and anisotropic $(M / H)_{\text {ani }}$ components were determined. The former can be obtained either from measured values for as-cast samples or from the average between the parallel $(M / H)_{\|}$and perpendicular $(M / H)$, values for stretched samples, and the latter from the differences between $(M / H)_{\text {iso }}$ and $(M / H)_{\|}$or $(M / H)_{\perp}$, i.e. $(M / H)_{\mathrm{ani}}=\left[(M / H)_{\|}-(M / H)_{\text {iso }}\right]$ or $\left[(M / H)_{\text {iso }}-(M / H)_{\perp}\right]$. Second, only a fraction $(\sim 60 \%)$ of the MWNTs powder exhibits alignment, so the measured $(M / H)_{\text {iso }}$ contains responses from all species in the powder, while $(M / H)_{\text {ani }}$ represents only the partially aligned portion. Since the multishell carbon species in the powder (about 30\%) exhibit smaller susceptibilities compared to MWNTs, ${ }^{4}$ and no significant amount of graphitic species are present, the measured isotropic mass susceptibility $(M / H)_{\text {iso }}$, which was obtained using the total mass of the powder, should be smaller than the intrinsic value of MWNTs. The measured anisotropic mass susceptibility $(M / H)_{\text {ani }}$, on the other hand, is about $60 \%$ of its intrinsic value, since only $\sim 60 \%$ of the powder contributed to the measured responses. Finally, the effect of angular distribution of the aligned MWNTs was deconvoluted using the distribution function $P(\phi)$ obtained from $x$-ray azimuthal $\phi$ scans [e.g., Fig. 1(b)] and by assuming the intrinsic susceptibility $\chi$ having the form ${ }^{2}$

$$
\chi=\chi_{\text {iso }}+\chi_{\text {ani }} \cos 2 \theta,
$$

where $\chi_{\text {iso }}$ is the isotropic component and $\chi_{\text {ani }}$ the anisotropic one, and $\theta$ is the polar angle with respect to the axis of the nanotube. Therefore, $\chi_{\|}=\chi_{\text {iso }}+\chi_{\text {ani }}$ and $\chi_{\perp}=\chi_{\text {iso }}-\chi_{\text {ani }}$. The presence of an angular distribution gives rise to a further correction to the observed anisotropic component $(M / H)_{\text {ani }}$ from $\chi_{\text {ani }}$. For a Gaussian distribution $P(\phi)$ with a relatively narrow width of $\Delta$, this correction $p(\Delta)$ is roughly $\exp \left(-\Delta^{2}\right)$ [about 0.8 for a $\Delta$ of $0.5\left(\sim 40^{\circ}\right)$ ]. Therefore the measured $(M / H)_{\text {ani }}$ is approximately $0.6 p(\Delta) \chi_{\text {ani }}$ or about $0.5 \chi_{\text {ani }}$. The isotropic values shown in Fig. 4 are the measured $(M / H)_{\text {iso, }}$, whose value at $300 \mathrm{~K}$ is about -2.5 $\times 10^{-6} \mathrm{emu} / \mathrm{g}$. Since these values underestimate the intrinsic behavior substantially, as indicated above, they are in good agreement with earlier measurements ${ }^{4-7}$ and predictions. ${ }^{2}$ Their temperature dependences are also in good agreement with earlier results. The anisotropic component shown in Fig. 4 is about $10^{-6} \mathrm{emu} / \mathrm{g}$, and nearly temperature independent. In other words, these estimates give values of -2 $\times 10^{-6}$ and $-3 \times 10^{-6} \mathrm{emu} / \mathrm{g}$ for $\chi_{\|}$and $\chi_{\perp}$, respectively. When compared to a previous estimate ${ }^{7}$ based on a model relating the magnetic susceptibility of graphite to that of the nanotubes with respective values of $-0.5 \times 10^{-6}$ and -11 $\times 10^{-6} \mathrm{emu} / \mathrm{g}$, the values obtained from this work appear to be larger than the above $\chi_{\|}$(parallel to the hexagon) and smaller than the $\chi_{\perp}$ (perpendicular to the hexagon).

In summary, we have studied the magnetic susceptibility of partially aligned MWNTs/polymer composites as a function of temperature and field. The intrinsic behavior of MWNTs has been estimated from measurements of a series of isotropic and aligned composites with different MWNT concentrations. The observed susceptibility is diamagnetic and anisotropic with the component along the tube less diamagnetic than the perpendicular counterpart. The observed behavior is in good agreement with theoretical predictions, but the anisotropy is opposite to previously reported measurements. While the causes of the discrepancies are not clear, further investigations with more controlled CNT samples, particularly with more pure and nearly monodispersed CNT, are necessary. Nevertheless this work with systematically aligned MWNTs represents an important first step to elucidating the magnetic behavior of CNT.

The work was supported in part by ONR N00014-98-10597 and NSF DMR-9601825 and DMR-9703419.

\footnotetext{
${ }^{1}$ S. Ijima, Nature (London) 354, 56 (1991).

${ }^{2}$ J. P. Lu, Phys. Rev. Lett. 74, 1123 (1995).

${ }^{3}$ H. Aijiki and T. Ando, J. Phys. Soc. Jpn. 62, 1255 (1993); 62, 2470 (1993)

${ }^{4}$ X. K. Wang, R. P. H. Chang, A. Patashinski, and J. B. Ketterson, J. Mater. Res. 9, 1578 (1994)

${ }^{5}$ A. P. Ramirez, R. C. Haddon, O. Zhou, R. M. Fleming, J. Zhang, S. M. McClure, and R. E. Smalley, Science 265, 84 (1994).

${ }^{6}$ P. Byszewski and M. Baran, Europhys. Lett. 31, 363 (1995).

${ }^{7}$ O. Chauvet, L. Forro, W. Bacsa, D. Ugarte, B. Doudin, and W. A. de Heer, Phys. Rev. B 52, 6963 (1995).

${ }^{8}$ L. Jin, C. Bower, and O. Zhou, Appl. Phys. Lett. 73, 1192 (1998)

${ }^{9}$ P. M. Ajayan, O. Stephen, C. Collier, and D. Trauth, Science 265, 1212 (1994).

${ }^{10}$ T. W. Ebbesen and P. M. Ajayan, Nature (London) 358, 16 (1992).
} 